\title{
Healthy eating index-2010 and physical activity and disabilities of old age
}

\author{
Martin M. Root*, Mark T. Nielsen, Timothy P. Smith and Katelyn M. Meaux \\ Department of Nutrition and Health Care Management, Appalachian State University, Boone, NC, USA
}

\begin{abstract}
.
BACKGROUND: Consuming a poor diet and being physically inactive may increase the risk of developing disabilities of old age.

OBJECTIVE: To investigate correlations between Healthy Eating Index-2010 (HEI-2010) scores, physical activity levels, and disabilities of old age in a large biracial cohort.

METHODS: The ARIC Study is a prospective cohort aged 45-64 years at baseline. Overall diet quality was assessed using the HEI-2010. A physical activity score was the sum of work, sports, and leisure. Activities of daily living (ADL), instrumental activities of daily living (IADL), and functional abilities were compared with HEI-2010 and physical activity by logistic regression 9 years after the baseline examination.

RESULTS: Compared with the lowest quartile, quartile 4 healthier HEI-2010 scores showed an odds ratios of 0.76 (95\% confidence interval 0.67-0.87) for functional disability, 0.89 (95\% CI 0.75-1.07) for ADL disabilities, and 0.88 (95\% CI 0.76-0.1.03) for IADL disabilities. Compared with the lowest tertile, tertile 3 of physical activity showed an odds ratios of 0.60 (95\% CI 0.54-0.67) for functional disability, 0.68 (95\% CI 0.60-0.78) for ADL disabilities, and 0.53 (95\% CI 0.47-0.60) for IADL disabilities.

CONCLUSIONS: Healthier diet and higher physical activity were associated with lower levels of the disabilities of aging, particularly functional disability, at follow up.
\end{abstract}

Keywords: Healthy diet, exercise, activities of daily living, aging

\section{Introduction}

Americans aged 65 years and older are the fastest growing segment of the US population. Since 1900, the percentage of this age group has more than tripled when compared to the total population [1]. In addition, the elderly are living longer than ever; persons living to age 65 have an average life expectancy of 18.8 more years, up from 14.7 years in 1950 . Unfortunately, physical and cognitive function typically decreases in older age. These functions may be assessed as activities of daily living (ADLs) and instrumental activities of daily living (IADLs). ADLs include daily self-care activities, while IADLs are those activities that go beyond basic functioning and allow individuals to live independently [2].

${ }^{*}$ Corresponding author: Martin M. Root, ASU Box 32168, Boone, NC 28608, USA. Tel.: +1 828262 2064; Fax: +1 828 262 8626; E-mail: rootmm@appstate.edu.
Maintaining independence, specifically independence in physical functioning, was one of the most commonly referenced factors when adults aged 90-95 years were asked to define "successful aging" [3]. Individuals with low physical functionality scores, similar to ADLs, have a significantly higher risk of all-cause mortality, and are more likely to be hospitalized [4]. In a nationwide study related to healthcare, individuals with limitations in ADLs and IADLs utilized outpatient services, emergency services, and in-patient services more often than those without limitations. Patients in the same study, specifically those with moderate to severe IADL limitations, could expect to spend two to three times as much money on total medical services [5]. Hardy et al. also showed that those who reported difficulty walking $1 / 4$ mile paid $\$ 2,773$ more in average total healthcare expenditures annually. The collateral economic costs associated with this type of limited mobility total over $\$ 42$ billion, and over 2 million 
additional hospitalizations [6]. While little may be possible to prevent these costs or reverse these disabilities once in place, preventive measures taken years and decades prior to onset have long been recognized as successful and cost effective.

Key factors leading to decreased functional abilities, ADLs, and IADLs have been investigated. Martin and Schoeni found that those with higher levels of education typically experience lower rates of disabilities, while those with increased obesity often experience higher rates of physical disabilities [7]. Seeman et al. showed that overweight and obese individuals displayed greater increases in reported IADL disabilities than those in the normal weight category [8]. This same study also found that nonHispanic Blacks, aged 60 to 69 years, reported significantly greater increases in ADL disabilities than non-Hispanic Whites. Factors such as low grip strength, low walking speed, and low physical activity throughout one's life have also been identified as strong predictors of ADL disabilities later in life [9]. In a recent meta-analysis, Tak et al. summarized physical activity studies examining the subsequent ADL disability. They found a consistently lower risk of ADL disability among those with higher initial physical activity levels [10].

Diet also has been shown to play a crucial role in prevention of physical disabilities, as well as lower rates of ADL and IADL- related disabilities. Based on the ARIC study of Blacks and Whites, Houston et al. found that higher vegetable, fruit, and dairy intakes were inversely associated with functional disability development [11]. Similarly, Kim et al. found that subjects following a Modified Traditional Korean diet, characterized by high consumption of fruits, dairy, and legumes, experienced a lower likelihood of ADL disability $(\mathrm{OR}=0.17,95 \%$ confidence interval [CI]: 0.05-0.56) [12]. Another similar study found the Mediterranean Diet (dense in vegetables, fruits, legumes, cereals, and fish) corresponded with decreased ADL and IADL disabilities [13]. This indicates that a healthy diet, rich in fruits, vegetables, and legumes, and even modest physical exercise may significantly alter outcomes related to improved aging outcomes, quality of life, and medical cost outcomes.

Updated every five years, the Healthy Eating Index (HEI) is a measure of diet quality derived from the recommendations of the Dietary Guidelines for Americans. In the past, the HEI has been applied to the evaluation of diet quality in subjects with diabetes [14], in cardiovascular risk [15], and in lung function in humans [16]; HEI-2005 scores have even been used to relate diet to symptoms of depression [17]. Xu et al. found that the odds of experiencing IADL disability, poor lower extremity mobility, and general physical activity disabilities were significantly lower in subjects who met the recommendations for HEI-2005 total fruit/whole fruit scores, compared with those who did not [18].

Limited research has been done to investigate the relationship between overall dietary quality, physical activity, and the prospective development of functional disabilities in old age. The ARIC study is a large prospective, bi-racial, middle-aged cohort. The objective of this research was to confirm a positive association between baseline HEI-2010 scores and physical activity scores and the development of positive functional outcomes at a nine-year followup as measured by ADL, IADL, and functional abilities.

\section{Methods}

\subsection{Subjects}

This manuscript was prepared using Atherosclerosis Risk in Communities (ARIC) Research Materials obtained from the National Heart Lung Blood Institute (NHLBI) Biologic Specimen and Data Repository Information Coordinating Center and does not necessarily reflect the opinions or views of the ARIC research groups or the NHLBI. The Institutional Review Board of Appalachian State University approved the acquisition and use of this dataset. The ARIC study is a large biracial, prospective cohort that aimed to investigate atherosclerosis and cardiovascular disease in four US communities [19]. Baseline data from 1987 through 1989 were available for 14,950 subjects. Subjects between the ages of 45 to 64 years were evaluated at 3-year intervals for four visits: 1987-1989, 1990-1992, 1993-1995, and 1996-1998.

Subjects who were identified during the initial physical examination as needing a wheel chair, using a cane, walking with an abnormal gait, having noticeable arm weakness, or having noticeable balance issues were excluded from the study. (Rhomberg test). Subjects who identified themselves as experiencing a prior stroke or heart failure, declared themselves to be in poor health, or identified themselves with chronic obstructive pulmonary disease were also excluded from the study. See Fig. 1. 


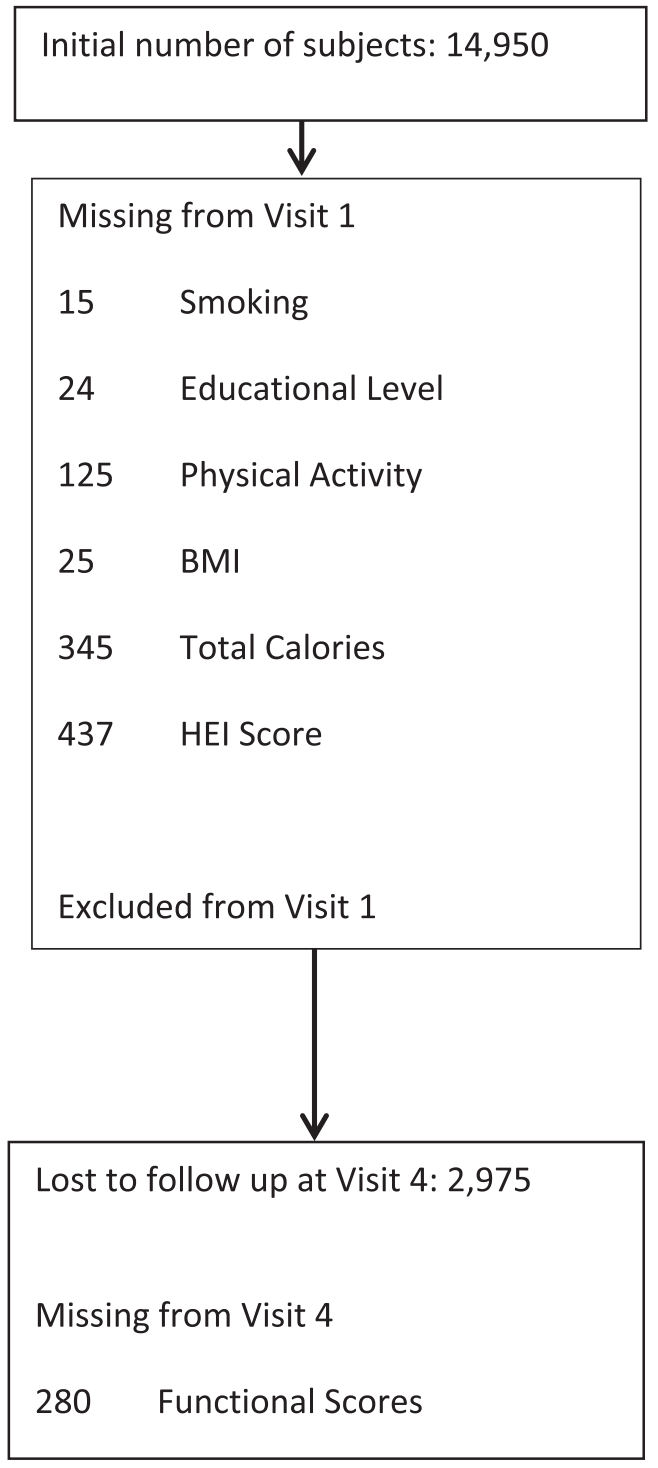

Fig. 1. Creation of the final study cohort.

\subsection{Functional limitations and disability}

Functional limitations and disabilities were grouped into three categories: activities of daily living (ADLs), instrumental activities of daily living (IADLs), and functional abilities. ADLs are considered the most essential of daily activities. These include getting dressed, walking from one room to another on the same floor, getting into and out of bed, holding a fork and cutting food, and drinking from a glass [20]. IADLs include completing chores around one's home, managing money, and preparing meals $[2,21]$. Functional ability tasks are the most physically demanding of the three categories. These include walking a quarter of a mile $(400 \mathrm{~m})$, standing from an armless chair, kneeling or stooping, and lifting and carrying a 10-pound $(4.5 \mathrm{~kg})$ load [21, 22].

During visit 4, 9 years after the initial visit, these abilities were evaluated by the Physical Ability Questionnaire containing 12 items grouped into the three categories above. In the questionnaire, subjects indicated the level of difficulty in completing tasks. Abilities were divided into a no impairment category (experiences no difficulty completing task) and an impaired category (including three stages: some difficulty, much difficulty, or unable to do). If any of the variables within each category were rated as impaired, then the subject was considered impaired for that category. This created 3 dichotomous variables for these abilities.

\subsection{Dietary analysis}

Participants were interviewed about their typical dietary intakes over the past year with a revised version of the semi-quantitative food frequency questionnaire validated by Willet et al. in the Nurses' Health Study [23]. Based on these results, nutrient composition of subjects' diets were calculated using the consumption frequency of each food, as well as its nutrient composition from the US Department of Agriculture Nutrient Composition Table [24, 25].

The Healthy Eating Index-2010 is a scoring method used to measure adherence to the Dietary Guidelines for Americans in 2010 [26, 27]. For this study, the HEI-2010 scoring was calculated using data from the ARIC food frequency questionnaire and nutrient derived data sets. A minor change was required in calculating the fatty acid HEI component score to make up for differences seen between optimal data required for the HEI-2010 and available data gathered from the ARIC food frequency questionnaire. The component score was calculated using vegetable oil (grams per day) from the nutrient data rather than the sum of mono and poly unsaturated fatty acids. The HEI-2010 was assessed by way of quartiles from lowest to highest. Higher scores are healthier.

\subsection{Physical activity and other measures}

Physical activity in ARIC was assessed according to a modified version of the Baecke survey [28]. Slight modifications were made to accommodate updates 
and to broaden some questions [29]. The results of the questionnaire were reduced to 3 scores $(1-5)$ for level of physical activity at work, during sports, and at leisure. Reliability (test-retest) over 6 years in the ARIC cohort ranged from $r=0.44$ to 0.53 [29]. Validity when comparing the sum of the 3 Baecke scores with doubly labelled water-assessed physical activity was $r=0.69$ [30]. For this analysis, these 3 scores were combined for a summed score of physical activity (1-15). Tertiles were then created from lowest to highest. A combined Baecke or Baecke-like score has been used in assessing overall physical activity in previous analyses [31-35]. Combining the work index with the more commonly used sport index is more inclusive of the type of physical activity experienced by Blacks and those with lower educational achievement in this cohort.

Height and weight were measured in the clinic at baseline and body mass index (BMI) was calculated. Interviewers also collected information related to multiple covariates such as age, sex, ethnicity, smoking habits, and level of education. Current diagnoses of coronary heart disease, heart failure, diabetes, and hypertension were also assessed during the interviews at visit 1 .

\subsection{Statistical analysis}

Statistics were performed with SPSS v. 22. Comparisons between subject characteristics across quartiles of HEI-2010 were performed with chisquare for nominal variables and ANOVA for continuous variables. Multivariate logistic regression was performed with the 3 measures of disability of old age as dichotomous outcomes. A simple model included only age (as a continuous variable), sex, and ethnicity (Black or non-Black) as independent variables. Multivariate model \#1 used in this analysis also included BMI, education (3 levels: less than high school degree, high school degree and vocational, college degree and more), total calories per day, and current smoking status (current smoker or not). Multivariate model \#2 also included the physical activity tertiles. The relation between the physical activity score tertiles and the disabilities of old age was assessed with the same multivariate models as above. Interactions between key independent variables and HEI quartiles were determined. P-trends was estimated from the ordered relationships of the odds ratios of disabilities across the quantiles of HEI2010 and physical activity. Sensitivity analysis was performed by repeating the primary analyses of HEI2010 and physical activity while eliminating key subgroups with pre-existing disease conditions. In a separate analysis, initially excluded subjects with a prior stroke, heart failure, poor health, or chronic obstructive pulmonary disease were returned to the cohort for sensitivity analysis.

\section{Results}

Figure 1 shows the selection process for subjects in the study. Given the number of missing values and the number excluded, there were a total of 12,343 subjects available for analysis from visit 1 . However, 2,975 were lost to follow up at visit 4 (9 years later) and 280 were missing information on functional abilities. This left 9,088 for the subsequent analyses.

Table 1 shows the characteristics of study subjects by quartiles of HEI-2010 in the ARIC study cohort from visit 1 including the covariates used in the later analyses. All parameters were significantly different across quartiles of HEI score. The change across quartiles was particularly notable for sex, current smokers, and education level.

At visit 4 there were 1,121 cases of ADL disabilities (12\% of the study population), 1,482 cases of IADL disabilities (17\%) and 4,277 cases of functional disability (47\%). In Table 2, the age-sex-ethnicity adjusted Simple models for ADL and IADL significantly decreased across quartiles of HEI-2010; this effect became attenuated, but retained statistical significance in model 1 . When additionally controlling for physical activity, the relationship of HEI-2010 and ADL and IADL become non-significant. However, the risk of functional disability remained substantially lower at higher quartiles of HEI-2010 across all 3 models.

The risk of the three disabilities of old age decreased considerably with level of initial physical activity (see Table 3 ). These were all improved about a third or better across the tertiles of combined physical activity even after controlling for the effect of HEI-2010. There was no significant interaction between HEI-2010 and physical activity.

For sensitivity testing, analysis subjects with coronary heart disease, hypertension, or diabetes at visit 1 were removed from the study one condition at a time. No significant changes were found in the primary results of HEI-2010 or physical activity. Adding back subjects with poor health or disabling chronic 
Table 1

Characteristics of study subjects by quartiles of HEI-2010 in ARIC study cohort

\begin{tabular}{|c|c|c|c|c|c|c|}
\hline \multirow[t]{2}{*}{ Variable } & \multirow[b]{2}{*}{$1-4$} & \multicolumn{4}{|c|}{ Quartile of HEI-2010 } & \multirow[t]{2}{*}{$P$} \\
\hline & & 1 & 2 & 3 & 4 & \\
\hline $\mathrm{N}$ & 9088 & 2272 & 2272 & 2272 & 2272 & \\
\hline HEI Score & 61 & 47 & 58 & 66 & 77 & \\
\hline (median \& range) & $(22-95)$ & $(22-51)$ & $(51-61)$ & $(61-70)$ & $(70-95)$ & \\
\hline Black $(\%)$ & 19 & 19 & 21 & 18 & 16 & 0.001 \\
\hline Female $(\%)$ & 56 & 35 & 49 & 61 & 71 & $<0.001$ \\
\hline Age (years) & $53.8 \pm 5.6$ & $52.7 \pm 5.5$ & $53.6 \pm 5.5$ & $54 \pm 5.7$ & $55 \pm 5.8$ & $<0.001$ \\
\hline Current Smoker (\%) & 20 & 31 & 23 & 17 & 12 & $<0.001$ \\
\hline Total Energy (Kcal) & $1624 \pm 596$ & $1947 \pm 656$ & $1718 \pm 568$ & $1533 \pm 517$ & $1294 \pm 425$ & $<0.001$ \\
\hline Total Energy $(\mathrm{kJ})$ & & $465 \pm 157$ & $411 \pm 136$ & $366 \pm 124$ & $309 \pm 102$ & $<0.001$ \\
\hline BMI $\left(\mathrm{kg} / \mathrm{m}^{2}\right)$ & $27.4 \pm 5.0$ & $27.4 \pm 4.9$ & $27.3 \pm 4.9$ & $27.4 \pm 5.3$ & $26.9 \pm 5.2$ & $<0.001$ \\
\hline Education $(\% \geq$ college $)$ & 41 & 31 & 41 & 44 & 47 & $<0.001$ \\
\hline Activity Level score & $7.1 \pm 1.4$ & $7.0 \pm 1.3$ & $7.1 \pm 1.4$ & $7.2 \pm 1.4$ & $7.3 \pm 1.4$ & $<0.001$ \\
\hline
\end{tabular}

Discrete variables were compared by Chi-Square. Continuous variables were compared by ANOVA.

Table 2

Characteristics of study subjects by tertiles of physical activity score in ARIC study cohort

\begin{tabular}{|c|c|c|c|c|c|}
\hline \multirow[t]{2}{*}{ Variable } & \multirow[b]{2}{*}{$1-3$} & \multicolumn{3}{|c|}{ Tertiles of physical activity score } & \multirow[t]{2}{*}{$P$} \\
\hline & & 1 & 2 & 3 & \\
\hline $\mathrm{N}$ & 9088 & 2771 & 3172 & 3145 & \\
\hline $\begin{array}{l}\text { Activity Level score (median } \\
\& \text { range) }\end{array}$ & $7.13(3.00-13.75)$ & $5.75(3.00-6.38)$ & $7.00(6.50-7.63)$ & $8.50(7.75-13.75)$ & \\
\hline Black $(\%)$ & 19 & 26 & 19 & 13 & $<0.001$ \\
\hline Female $(\%)$ & 56 & 67 & 56 & 46 & $<0.001$ \\
\hline Age (years) & $53.8 \pm 5.6$ & $52.7 \pm 5.5$ & $53.6 \pm 5.5$ & $54 \pm 5.7$ & $<0.001$ \\
\hline Current Smoker (\%) & 20 & 22 & 20 & 18 & $<0.001$ \\
\hline Total Energy (Kcal) & $1624 \pm 596$ & $1575 \pm 580$ & $1610 \pm 584$ & $1682 \pm 617$ & $<0.001$ \\
\hline Total Energy (kJ) & $388 \pm 142$ & $376 \pm 139$ & $385 \pm 140$ & $402 \pm 147$ & $<0.001$ \\
\hline BMI $\left(\mathrm{kg} / \mathrm{m}^{2}\right)$ & $27.4 \pm 5.0$ & $27.9 \pm 5.4$ & $27.4 \pm 5.0$ & $28.8 \pm 4.5$ & $<0.001$ \\
\hline Education ( $\% \geq$ college) & 41 & 31 & 41 & 44 & $<0.001$ \\
\hline HEI 2010 Score & $60 \pm 13$ & $59 \pm 13$ & $60 \pm 13$ & $62 \pm 13$ & $<0.001$ \\
\hline
\end{tabular}

Discrete variables were compared by Chi-Square. Continuous variables were compared by ANOVA.

Table 3

Odds ratios of disabilities of old age by quartiles of HEI-2010 in the ARIC cohort

\begin{tabular}{|c|c|c|c|c|c|c|c|}
\hline \multirow[t]{2}{*}{ Disability } & \multirow{2}{*}{$\begin{array}{l}\text { Disability } \\
\text { (percent) }\end{array}$} & \multirow[t]{2}{*}{ Model } & \multicolumn{4}{|c|}{ Quartiles of HEI-2010 } & \multirow{2}{*}{$\begin{array}{c}\text { Trend } \\
P \text {-value }\end{array}$} \\
\hline & & & $\overline{1}$ & 2 & 3 & 4 & \\
\hline \multirow[t]{3}{*}{ Functional } & 47 & Simple & 1.00 & $0.81(0.72-0.92)^{* * *}$ & $0.68(0.60-0.78)^{* * *}$ & $0.58(0.52-0.66)^{* * *}$ & $<0.001$ \\
\hline & & Multivariate 1 & 1.00 & $0.87(0.77-0.98)^{*}$ & $0.75(0.66-0.76)^{* * *}$ & $0.70(0.62-0.80)^{* * *}$ & $<0.001$ \\
\hline & & Multivariate 2 & 1.00 & $0.89(0.79-1.00)$ & $0.78(0.68-0.89)^{* * *}$ & $0.76(0.67-0.87)^{* * *}$ & 0.001 \\
\hline \multirow[t]{3}{*}{$\mathrm{ADL}$} & 12 & Simple & 1.00 & $0.79(0.67-0.94)^{* *}$ & $0.78(0.65-0.91)^{* *}$ & $0.67(0.57-0.78)^{* * *}$ & $<0.001$ \\
\hline & & Multivariate 1 & 1.00 & $0.86(0.74-1.01)$ & $0.89(0.76-1.06)$ & $0.84(0.71-1.00)^{*}$ & 0.013 \\
\hline & & Multivariate 2 & 1.00 & $0.89(0.76-1.04)$ & $0.91(0.77-1.10)$ & $0.89(0.75-1.07)$ & 0.057 \\
\hline \multirow[t]{3}{*}{ IADL } & 17 & Simple & 1.00 & $0.81(0.70-0.93)^{* *}$ & $0.72(0.62-0.84)^{* * *}$ & $0.65(0.56-0.73)^{* * *}$ & $<0.001$ \\
\hline & & Multivariate 1 & 1.00 & $0.88(0.77-1.02)$ & $0.82(0.72-0.95)^{* *}$ & $0.79(0.68-0.92)^{* *}$ & 0.012 \\
\hline & & Multivariate 2 & 1.00 & $0.92(0.80-1.05)$ & $0.87(0.75-1.02)$ & $0.88(0.76-1.03)$ & 0.170 \\
\hline
\end{tabular}

Simple logistic model covariates are age, sex, and ethnicity (Black or non-Black). Multivariate 1 model covariates are simple covariates and BMI, education (3 levels), total calories per day, and current smoking status (current smoker or not). Multivariate 2 model includes the Multivariate 1 variables and quartiles of physical activity. ${ }^{*} p<0.05,{ }^{* *} p<0.01,{ }^{* * *} p<0.001$ when compared to Quartile 1 . 
Table 4

Odds ratios of disabilities of old age by tertiles of physical activity in the ARIC cohort

\begin{tabular}{lccccc}
\hline Disability & Model & \multicolumn{3}{c}{ Tertiles of Physical Activity } & Trend \\
\cline { 3 - 5 } & & 1 & 2 & 3 & $P$-value \\
\hline Functional & simple & 1.00 & $0.70(0.64-0.77)^{* * *}$ & $0.56(0.50-0.61)^{* * *}$ & $<0.001$ \\
& Multivariate 1 & 1.00 & $0.72(0.65-0.80)^{* * *}$ & $0.59(0.53-0.66)^{* * *}$ & $<0.001$ \\
& Multivariate 2 & 1.00 & $0.73(0.65-0.80)^{* * *}$ & $0.60(0.54-0.67)^{* * *}$ & $<0.001$ \\
ADL & simple & 1.00 & $0.65(0.57-0.74)^{* * *}$ & $0.63(0.56-0.72)^{* * *}$ & $<0.001$ \\
& Multivariate 1 & 1.00 & $0.67(0.59-0.80)^{* * *}$ & $0.68(0.59-0.77)^{* * *}$ & 0.002 \\
& Multivariate 2 & 1.00 & $0.66(0.59-0.76)^{* * *}$ & $0.68(0.60-0.78)^{* * *}$ & $<0.001$ \\
IADL & simple & 1.00 & $0.62(0.56-0.69)^{* * *}$ & $0.50(0.45-0.56)^{* * *}$ & $<0.001$ \\
& Multivariate 1 & 1.00 & $0.64(0.57-0.72))^{* * *}$ & $0.53(0.47-0.59)^{* * *}$ & $<0.001$ \\
& Multivariate 2 & 1.00 & $0.64(0.57-0.72)^{* * *}$ & $0.53(0.47-0.60)^{* * *}$ & $<0.001$ \\
\hline
\end{tabular}

Simple logistic model covariates are age, sex and ethnicity (Black or non-Black). Multivariate model 1 covariates are simple covariates and BMI, education (3 levels), total calories per day, and current smoking status (current smoker or not). Multivariate model 2 are model 1 covariates and quartiles of HEI-2010. $* * *<0.001$ when compared to Tertile 1.

conditions also did not materially alter the results. No substantive interactions were found in the primary results with age groups, sex, ethnicity groups, smoking subgroups, BMI levels, or education levels.

\section{Discussion}

This study suggests that there is a significant inverse association between quality of diet as measured by HEI-2010 and functional disability, and also between the level of overall physical activity and the increasing disabilities of old age, including functional disabilities, ADL, and IADL.

Dietary effects on subsequent disabilities of old age have been found by other investigators. $\mathrm{Xu}$ et al. found that adherence to the Dietary Guidelines for Americans 2005 was associated with lower disability in physical, social, and psychological domains in older adults [18]. Kim et al. determined that individuals following a Modified Traditional Korean diet containing higher amounts of fruit, dairy, and legumes, experienced a lower likelihood of developing ADL disability when compared to a carbohydrate-dense Traditional Korean eating pattern [12]. Houston et al. found that higher amounts of vegetables, fruits, and dairy in the diet were inversely associated with functional limitations in men and women of the ARIC study [11]. Feart et al. showed that women with higher adherence to the Mediterranean diet were less likely to experience IADL disabilities as they age [13]. Milaneschi et al. reviewed recent studies on nutrition and mobilityrelated issues in older adults. According to this review, current evidence now supports that diets high in fruits and vegetables, such as the Mediterranean Diet, play a valuable role in aging [36]. Diets higher in fruits, vegetables, soy products, and fish and lower in soft drinks, alcoholic beverages, and rice (which would be measured with a high HEI-adherence score) were found to have protective effects on the cognition of older Japanese adults and were correlated with lower incidences of dementia [37]. The present study found only limited association between a diet and lower ADL and IADL and these associations were attenuated in the full models that included physical activity. Indeed, after controlling for age, sex, and ethnicity, there is a strong correlation between HEI-2010 score and physical activity score $(\mathrm{R}=0.138)$.

Physical activity has consistently been shown to predict better physical, more functional, and less disabled outcomes later in life [10]. It is interesting to note that even modest physical activity, as when comparing tertile 2 with tertile 1 in this study's results, is correlated with significantly lower risk of all 3 disabilities. Even with the use of a very general physical activity score, these consistent correlations were noted. No effort was made to distinguish specific types of activity that were more beneficial over others. Martin et al. have confirmed that the early stages of disease contribute to multiple trajectories of disability [38]. Poher et al found that physical activity programs were more effective in preventing disability in adults between the ages of 70 and 89 years than a health education program alone [39]. Chodzko-Zajko et al. suggest the importance of physical activity in delaying the earliest stages of this process during aging [40]. In addition to the direct effects of physical activity, indirect effects such as improved self-efficacy and reduced depression may also impact the risk of 
disabilities of old age. Depression has been found to negatively influence the level of physical activity and subsequently increase the risk of disability [41]. Finally, unknown or unmeasured confounding variables may lead to overestimation of the correlation between physical activity and abilities of old age.

Dietary Guidelines for Americans 2010 is characterized by high intakes of fruits, vegetables, whole grains, low-fat dairy, lean meats, and seafood. A diet that is high in fruits and vegetables is likely to contain higher levels of phytochemical antioxidants. These phytochemical and vitamin antioxidants can counter oxidative stress in older adults, which may reduce levels of inflammation and neurological effects, which may affect ADLs, IADLs, and functional abilities. It is proposed that serum antioxidants are critical in reducing the inflammatory response caused by increased release of cytokine interleukin6 in the blood, [42] which have been linked to poor health outcomes in older adults [43]. Gianni et al. found that there is an increase in oxidative damage to DNA and proteins, particularly in muscle, as people age which potentially contributes to sarcopenia [44]. Sarcopenia contributes to the development of physical disabilities of old age. Cesari et al. also determined a correlation between higher blood concentration of vitamin antioxidants and increased physical ability, particularly muscle strength [45]. In addition, low levels of serum carotenoids have been associated with decreased grip, hip, and knee strength, all of which are of particular importance for older adults [46]. Neuroinflammation in response to dietary factors may also play a role in neurodegenerative diseases and possibly a decline in ADLs and IADLs [47, 48].

The ARIC cohort can be compared with other groups from the US of about the same age for their diet and disabilities. The older adults (age $>60$ ) participating in the 1999-2002 National Health and Nutrition Examination Survey (NHANES) had an average HEI score of only 66.6 out of 100 , with $>80$ defined as a recommended diet. Recommendations for fruits, vegetables, and dairy were met by less than one-third of this group [49]. Our ARIC cohort had an average score of 62 with for an age range at visit 1 of 45-64. This was a somewhat lower score in a younger group than from NHANES. Comparing ADL and IADL with other studies was a bit more difficult. There was a range of definitions of these disabilities. A recent MMWR [50] article matched 3 of the 5 indicators for ADL in this report and 2 of 3 indicators for IADL. They reported a disability prevalence of about $2-3 \%$ for ADL and $4-6 \%$ for
IADL for a similar age range. In contrast, Hung et al with a similar comparability to our indicators found disabilities of $19 \%$ for ADL and $18 \%$ for IADL in a comparably aged group [51]. Our results were 12\% ADL and 17\% IADL disabilities.

No interaction was noted between the two lifestyle factors we considered (diet and physical activity) when considering disabilities of aging. While not considering physical disability directly, it is interesting to note a recent study by Nijholt et al. found no synergistic interaction in the beneficial roles of diet and exercise associated with cognitive function of old age [52].

There were limitations to this study. These results cannot conclude causation because ARIC was an epidemiological observational study. Since the ARIC study was performed using a bi-racial cohort, results cannot be associated with races other than White or Black. As described above, the preferred exclusion of subjects with functional, ADL, and IADL disabilities at visit 1 was not possible because these data were not collected at that time. Variables that were available concerning physical limitations were utilized for this purpose. The substitution of vegetable oil for mono and poly unsaturated fats would tend to increase this fatty acid component score slightly due to the presence of other fatty acids in the vegetable oil sum. This would tend to make this population appear to have a slightly healthier diet than they were in actuality. Physical activity could not be assessed directly, but was instead assessed as a score that reflected selfdeclared activity levels at work, leisure, and sport. Understandably, those who were unable to participate in physical activities due to disability would score low in the original assessment and also would be low in the disabilities of age assessment nine years later. Subjects who declared a disability or condition that might reduce ability were eliminated from this study in an effort to minimize this effect. These exclusions were very similar to those by Houston et al. in their study on diet and disabilities in the ARIC cohort [11]. During sensitivity analysis, these exclusions were selectively increased to observe their potential effects on the results. Only modest effects were noted. Physical activity was found to attenuate dietary effects, though there was not a significant interaction between the two.

\section{Conclusions}

A better diet characterized by adherence the Dietary Guidelines for Americans 2010 was found to 
be significantly associated sustained functional abilities. Higher levels of physical activity were also associated with improved subsequent abilities of aging including functional abilities, ADL, and IADL.

\subsection{Take away points}

- Disabilities increase with aging, including functional disabilities, Activities of Daily Living disabilities, and Independent Activities of Daily Living disabilities.

- Overall, closer adherence to the Dietary Guidelines for Americans 2010 throughout middle age may reduce functional disabilities in older years.

- Staying physically active whether on the job, in sports, or at leisure may also significantly reduce the onset of these disabilities.

\section{Acknowledgments}

We acknowledge the editorial help of Alexandria Harrell for final edits and rewrites of this manuscript. There was no external support for this publication. The authors have no conflicts of interest to report.

\section{References}

[1] Bernstein M, Munoz N. Position of the Academy of Nutrition and Dietetics: Food and nutrition for older adults: Promoting health and wellness. J Acad Nutr Diet. 2012;112(8):1255.

[2] Lawton MP, Brody EM. Assessment of older people: Selfmaintaining and instrumental activities of daily living. Gerontologist. 1969;9(3):179.

[3] Nosraty L, Jylha M, Raittila T, Lumme-Sandt K. Perceptions by the oldest old of successful aging, Vitality 90+ Study. J Aging Stud. 2015;32:50.

[4] Legrand D, Vaes B, Mathei C, Adriaensen W, Van Pottelbergh G, Degryse JM. Muscle strength and physical performance as predictors of mortality, hospitalization, and disability in the oldest old. J Am Geriatr Soc. 2014;62(6):1030.

[5] Wu CY, Hu HY, Li CP, Fang YT, Huang N, Chou YJ. The association between functional disability and acute care utilization among the elderly in Taiwan. Arch Gerontol Geriatr. 2013;57(2):177.

[6] Hardy SE, Kang Y, Studenski SA, Degenholtz HB. Ability to walk $1 / 4$ mile predicts subsequent disability, mortality, and health care costs. J Gen Intern Med. 2011;26(2):130.

[7] Martin LG, Schoeni RF. Trends in disability and related chronic conditions among the forty-and-over population: 1997-2010. Disabil Health J. 2014;7(1 Suppl):S4.

[8] Seeman TE, Merkin SS, Crimmins EM, Karlamangla AS. Disability trends among older Americans: National Health and Nutrition Examination Surveys, 1988-1994 and 19992004. Am J Public Health. 2010;100(1):100.
[9] Vermeulen J, Neyens JC, van Rossum E, Spreeuwenberg MD, de Witte LP. Predicting ADL disability in communitydwelling elderly people using physical frailty indicators: A systematic review. BMC Geriatr. 2011;11:33.

[10] Tak E, Kuiper R, Chorus A, Hopman-Rock M. Prevention of onset and progression of basic ADL disability by physical activity in community dwelling older adults: A meta-analysis. Ageing Res Rev. 2013;12(1):329.

[11] Houston DK, Stevens J, Cai J, Haines PS. Dairy, fruit, and vegetable intakes and functional limitations and disability in a biracial cohort: The Atherosclerosis Risk in Communities Study. Am J Clin Nutr. 2005;81(2):515.

[12] Kim J, Lee Y, Lee SY, Kim YO, Chung YS, Park SB. Dietary patterns and functional disability in older Korean adults. Maturitas. 2013;76(2):160.

[13] Feart C, Peres K, Samieri C, Letenneur L, Dartigues JF, Barberger-Gateau P. Adherence to a Mediterranean diet and onset of disability in older persons. Eur J Epidemiol. 2011;26(9):747.

[14] Direktor S, Ozer E. Evaluating dietary quality in diabetes by the Healthy Eating Index. Asia Pac J Clin Nutr. 2013;22(4):620.

[15] Nicklas TA, O'Neil CE, Fulgoni VL, 3rd. Diet quality is inversely related to cardiovascular risk factors in adults. J Nutr. 2012;142(12):2112.

[16] Root MM, Houser SM, Anderson JJ, Dawson HR. Healthy Eating Index-2005 and selected macronutrients are correlated with improved lung function in humans. Nutr Res. 2014;34(4):277.

[17] Exebio JC, Zarini GG, Exebio C, Huffman FG. Healthy Eating Index scores associated with symptoms of depression in Cuban-Americans with and without type 2 diabetes: A cross sectional study. Nutr J. 2011;10:135.

[18] Xu B, Houston D, Locher JL, Zizza C. The association between Healthy Eating Index-2005 scores and disability among older Americans. Age Ageing. 2012;41(3):365.

[19] ARIC Investigators. The Atherosclerosis Risk in Communities (ARIC) Study: Design and objectives. Am J Epidemiol. 1989;129(4):687.

[20] Katz S, Ford AB, Moskowitz RW, Jackson BA, Jaffe MW. Studies of illness in the aged. The index of ADL: A standardized measure of biological and psychosocial function. JAMA. 1963;185:914.

[21] Rosow I, Breslau N. A Guttman health scale for the aged. J Gerontol. 1966;21(4):556.

[22] Nagi SZ. An epidemiology of disability among adults in the United States. Milbank Mem Fund Q Health Soc. 1976;54(4):439.

[23] Willett WC, Sampson L, Stampfer MJ, Rosner B, Bain C, Witschi J, Hennekens CH, Speizer FE. Reproducibility and validity of a semiquantitative food frequency questionnaire. Am J Epidemiol. 1985;122(1):51.

[24] Human Nutrition Information Service. Composition of foods Washington, DC: US Department of Agriculture; 1989 [Available from: http://naldc.nal.usda. gov/download/CAT91958298/PDF.

[25] Matthews RH, Pehrsson PR, Farhat-Sabet M. Sugar content of selected foods: Individual and total sugar. Washington, D.C.: U.S. Dept. of Agriculture, Human Nutrition Information Service; 1987.

[26] Guenther PM, Casavale KO, Reedy J, Kirkpatrick SI, Hiza HA, Kuczynski KJ, Kahle LL, Krebs-Smith SM. Update 
of the healthy eating index: HEI-2010. J Acad Nutr Diet. 2013;113(4):569.

[27] U.S. Department of Agriculture and U.S. Department of Health and Human Services. Dietary Guidelines for Americans, 2010. Washington, DC: U.S. Government Printing Office; 2010. Available from: http://www.health.gov/ dietaryguidelines/dga2010/DietaryGuidelines2010.pdf.

[28] Baecke JA, Burema J, Frijters JE. A short questionnaire for the measurement of habitual physical activity in epidemiological studies. Am J Clin Nutr. 1982;36(5):936.

[29] Folsom AR, Arnett DK, Hutchinson RG, Liao F, Clegg LX, Cooper LS. Physical activity and incidence of coronary heart disease in middle-aged women and men. Med Sci Sports Exerc. 1997;29(7):901.

[30] Westerterp KR. Assessment of physical activity level in relation to obesity: Current evidence and research issues. Med Sci Sports Exerc. 1999;31(11 Suppl):S522.

[31] Van Halewyck F, Lavrysen A, Levin O, Elliott D, Helsen WF. The impact of age and physical activity level on manual aiming performance. J Aging Phys Act. 2015;23(2):169.

[32] Steins Bisschop CN, Peeters PHM, Monninkhof EM, van der Schouw YT, May AM. Associations of visceral fat, physical activity and muscle strength with the metabolic syndrome. Maturitas. 2013;76(2):139.

[33] Van Halewyck F, Lavrysen A, Levin O, Boisgontier MP, Elliott D, Helsen WF. Both age and physical activity level impact on eye-hand coordination. Hum Mov Sci. 2014;36:80.

[34] Vancampfort D, De Herdt A, Vanderlinden J, Lannoo M, Soundy A, Pieters G, Adriaens A, De Hert M, Probst M. Health related quality of life, physical fitness and physical activity participation in treatment-seeking obese persons with and without binge eating disorder. Psychiatry Research. 2014;216(1):97.

[35] Mori T, Ishii S, Greendale GA, Cauley JA, Sternfeld B, Crandall CJ, Han W, Karlamangla AS. Physical activity as determinant of femoral neck strength relative to load in adult women: Findings from the hip strength across the menopause transition study. Osteopor Int. 2014;25(1):265.

[36] Milaneschi Y, Tanaka T, Ferrucci L. Nutritional determinants of mobility. Curr Opin Clin Nutr Metab Care. 2010;13:625.

[37] Okubo H, Inagaki H, Gondo Y, Kamide K, Ikebe K, Masui Y, Arai Y, Ishizaki T, Sasaki S, Nakagawa T, Kabayama M, Sugimoto K, Rakugi H, Maeda Y. Association between dietary patterns and cognitive function among 70-year-old Japanese elderly: A cross-sectional analysis of the SONIC study. Nutr J. 2017;16(1):56.

[38] Martin LG, Zimmer Z, Lee J. Foundations of activity of daily living trajectories of older americans. Journals of Gerontology Series B, Psychological Sciences and Social Sciences. 2015;72(1):129.

[39] Pahor M, Guralnik JM, Ambrosius WT, et al. Effect of structured physical activity on prevention of major mobility disability in older adults: The LIFE study randomized clinical trial. JAMA. 2014;311(23):2387.
[40] Chodzko-Zajko WJ, Proctor DN, Fiatarone Singh MA, Minson CT, Nigg CR, Salem GJ, Skinner JS. American college of sports medicine position stand. Exercise and physical activity for older adults. Med Sci Sports Exerc. 2009;41(7):1510.

[41] Penninx BW, Leveille S, Ferrucci L, van Eijk JT, Guralnik JM. Exploring the effect of depression on physical disability: Longitudinal evidence from the established populations for epidemiologic studies of the elderly. Am J Public Health (N Y). 1999;89(9):1346.

[42] Walston J, Xue Q, Semba RD, Ferrucci L, Cappola AR, Ricks M, Guralnik J, Fried LP. Serum antioxidants, inflammation, and total mortality in older women. Am J Epidemiol. 2006;163(1):18.

[43] Young AJ, Phillip DM, Lowe GM. Carotenoid antioxidant activity. In: Krinsky NI, Mayne ST, Sies H, editors. Carotenoids in Health and Disease. New York, NY: Marcel Dekker; 2004, p. 105.

[44] Gianni P, Jan KJ, Douglas MJ, Stuart PM, Tarnopolsky MA. Oxidative stress and the mitochondrial theory of aging in human skeletal muscle. Exp Gerontol. 2004;39(9):1391.

[45] Cesari M, Pahor M, Bartali B, Cherubini A, Penninx BW, Williams GR, Atkinson H, Martin A, Guralnik JM, Ferrucci L. Antioxidants and physical performance in elderly persons: The Invecchiare in Chianti (InCHIANTI) study. Am J Clin Nutr. 2004;79(2):289.

[46] Semba RD, Lauretani F, Ferrucci L. Carotenoids as protection against sarcopenia in older adults. Arch Biochem Biophys. 2007;458(2):141.

[47] Almeida S, Alves MG, Sousa M, Oliveira PF, Silva BM. Are polyphenols strong dietary agents against neurotoxicity and neurodegeneration? Neurotoxicity Research. 2016;30(3):345.

[48] Ozawa M, Shipley M, Kivimaki M, Singh-Manoux A, Brunner EJ. Dietary pattern, inflammation and cognitive decline: The Whitehall II prospective cohort study. Clin Nutr. 2016;36.

[49] Ervin RB. Healthy Eating Index scores among adults, 60 years of age and over, by sociodemographic and health characteristics: United States, 1999-2002. Advance data from vital and health statistics. no 395. Hyattsville, MD: National Center for Health Statistics; 2008.

[50] QuickStats: Percentage of adults aged $>/=45$ years with activity limitations, by age group and type of limitation - national health interview survey, United States, 2000-2015. MMWR. 2016;65(33):884.

[51] Hung WW, Ross JS, Boockvar KS, Siu AL. Recent trends in chronic disease, impairment and disability among older adults in the United States. BMC Geriatrics. 2011;11:47.

[52] Nijholt W, Jager-Wittenaar H, Visser M, Van Der Schans $\mathrm{CP}$, Hobbelen JSM. Are a healthy diet and physical activity synergistically associated with cognitive functioning in older adults? J Nutr Health Aging. 2016;20(5):525. 